\title{
pyHIVE, a health-related image visualization and engineering system using Python
}

\author{
Ruochi Zhang ${ }^{1}$, Ruixue Zhao ${ }^{1}$, Xinyang Zhao ${ }^{1}$, Di Wu' ${ }^{2}$, Weiwei Zheng ${ }^{1}$, Xin Feng ${ }^{1^{*}}$ and Fengfeng Zhou ${ }^{{ }^{*}}$ (DD
}

\begin{abstract}
Background: Imaging is one of the major biomedical technologies to investigate the status of a living object. But the biomedical image based data mining problem requires extensive knowledge across multiple disciplinaries, e.g. biology, mathematics and computer science, etc.

Results: pyHIVE (a Health-related Image Visualization and Engineering system using Python) was implemented as an image processing system, providing five widely used image feature engineering algorithms. A standard binary classification pipeline was also provided to help researchers build data models immediately after the data is collected. pyHIVE may calculate five widely-used image feature engineering algorithms efficiently using multiple computing cores, and also featured the modules of Principal Component Analysis (PCA) based preprocessing and normalization.

Conclusions: The demonstrative example shows that the image features generated by pyHIVE achieved very good classification performances based on the gastrointestinal endoscopic images. This system pyHIVE and the demonstrative example are freely available and maintained at http://www.healthinformaticslab.org/supp/resources.php.
\end{abstract}

\section{Background}

Besides OMIC data, imaging is another major source of biomedical information for the biomedical modeling [1]. And imaging has its inherent nature of non-invasively and instantly monitoring the health status inside the body [2], while the OMIC data is produced hours or longer later after the sample is collected. So imaging and OMIC data represent different modalities and resolution of a biological system [3].

Biomedical images have already been widely used in the diagnosis and prognosis modeling [4-6]. The image histogram of oriented gradients (HOG) feature was used for the predictions of lung cancers [7]. And the rotation-invariant local binary pattern (LBP) feature and its variants were utilized to investigate the texture and other image patterns in biomedical images [8-10]. Other image features like the gray-level co-occurrence matrix (GLCM) were also frequently utilized in predicting the tumor outcomes and other phenotypes [11]. Image segmentation, denoising and

\footnotetext{
* Correspondence: 573175490@qq.com; FengfengZhou@gmail.com 1 Bioknow Health Informatics Lab, College of Computer Science and Technology, and Key Laboratory of Symbolic Computation and Knowledge Engineering of Ministry of Education, Jilin University, Changchun 130012, Jilin, China

Full list of author information is available at the end of the article
}

fractal image features were essential to improve the image based classification problems [4-6].

This work presented a user-friendly system, pyHIVE, to extract five widely used image features using the Python programming language. The existing image feature extraction softwares usually focus on one algorithm, and the users need to implement a separate script to optimize the prediction performance of a disease classification model. pyHIVE closes the gap between the raw biomedical images and the standard input for the data mining researchers. pyHIVE provides five widely used image feature extraction algorithms and produces all the data matrices required for a standard data mining task. A demonstrative script is also provided for a PCA-based feature selection and binary classification example. A parallelization function is implemented for a better utilization of the widely-used multi-core computing environments. We hope that pyHIVE may help the researchers of interest intuitively ask data mining questions from the biomedical images, and facilitate the faster developments of image-based disease diagnosis and prognosis modeling.

(c) The Author(s). 2018 Open Access This article is distributed under the terms of the Creative Commons Attribution 4.0 International License (http://creativecommons.org/licenses/by/4.0/), which permits unrestricted use, distribution, and reproduction in any medium, provided you give appropriate credit to the original author(s) and the source, provide a link to the Creative Commons license, and indicate if changes were made. The Creative Commons Public Domain Dedication waiver (http://creativecommons.org/publicdomain/zero/1.0/) applies to the data made available in this article, unless otherwise stated. 


\section{Materials and methods Dataset details}

We demonstrated how to use pyHIVE by a public dataset of gastrointestinal endoscopic images. The endoscopic images are publicly available at the El Salvador Atlas of Gastrointestinal Video Endoscopy, as similar in [12]. 243 images were randomly captured from the 16 endoscopic videos of normal participants, and each is $1280 \times 720$ pixels in size. These were regarded as the negative images. There are 17 gastric polyp videos, 26 gastric ulcer videos and 10 gastritis videos available at the database. A random image capturing step generated 158, 99 and 74 images from these three groups of videos, respectively. These $331(=158+99+74)$ images were regarded as the positive images, and are $352 \times 240$ pixels in size. The negative images were scaled to the same size of the positive ones, which is a user-defined parameter of pyHIVE.

\section{Workflow and explanations of the pyHIVE code}

The software pyHIVE was designed as a parallel image feature engineering system, and the overall workflow was demonstrated in Fig. 1. Firstly, pyHIVE has a few prerequisite python packages, including numpy version 1.12.1, pandas version 0.19.2, Pillow version 4.1.0, scikit-image version 0.13 ., scikit-leran version 0.18 .1 , and scipy version 0.19.0. These python packages were pre-installed in many python distributions. But in case that these python packages were not installed, the user may manually install them using the python command "pip", e.g., pip install numpy.

After all the prerequisite python packages were available, the user may run pyHIVE by the command line "python main.py". pyHIVE loads all the image files in the sub-directory "images" by default, and the output files will be in the sub-directory "features". All the parameters of the five image feature engineering algorithms may be tuned in the configuration file "config.cof". Default values for all the parameters are also provided for the convenience of the users.

A task "Processor" will be executed on an input image file and image features will be calculated based on the user-specified algorithms and their parameters. An optional "Normalize" step is provided. All the calculated features will merged into one file for each image file. pyHIVE will parallelly run multiple "Processor" tasks based on the user-specified parameter "njob" in the configuration file config.cof. Feature files of all the images will be merged into one matrix file.

\section{Implementation}

pyHIVE implemented five widely-used image feature extraction algorithms, i.e. Histogram of Oriented Gradient (HOG), Local Binary Pattern (LBP), Gray-level
Co-occurrence Matrix (GLCM), Hessian Matrix (HEM) and Canny (CAN), using the programming language Python. HOG, LBP and GLCM are three widely-used image features to describe textures [7-9, 11]. HEM and CAN work well on object topology detections in images $[13,14]$. A post-processing module using Principal Component Analysis (PCA) was also provided. The eigenvalues of the aforementioned five algorithms generate different value scales, and the user may choose to normalize the data using an embedded normalization module.

pyHIVE also parallelized the calculations of the aforementioned image feature extraction algorithms. The calculation of image features has an inherent nature of $\mathrm{O}(n \times m)$ time complexity, where $n$ and $m$ are the width and height of an image, respectively. Some image feature extraction algorithms have been implemented using interpretive languages like MatLab, and may run for minutes or even hours to extract features of a large image. It may become intolerable in real-time analysis situations or for thousands of biomedical images. Python was chosen to implement pyHIVE for its fast running and a large repository of data mining modules. pyHIVE may fully utilize the computing power of multi-core architecture of the modern servers. Due to that the Windows operation system does not provide a strong support for the parallel programming interface in Python, the Windows version of pyHIVE does not support parallelized calculation. It has a time cost to switch between computing processes in the same CPU core, so it is recommended that the number of parallel tasks may be set to the number of CPU cores in the user server.

\section{Extensive support for input and output formats}

pyHIVE has been conceived and implemented as a user-friendly image feature extraction system. So pyHIVE has been tested using both Python v2 and Python $\mathrm{v} 3$ in all the three main operation systems, i.e. Linux, Mac OS and Windows. pyHIVE accepts 30+ image file formats as input, e.g. BMP, EPS, GIF, JPG, PNG, PPM, and SGI, etc. The calculated features may be saved as one or more choices of the six supported file formats, i.e. CSV, PICKLE, JSON, EXCEL, TXT and SQL. The user may decide the number of fractional digits of the decimal values of the calculated image features. A larger file size and a longer saving time will be needed if a larger number of fractional digits is chosen.

Due to the many parameters that a user may want to manipulate, pyHIVE defined a configuration file to help the user tune how pyHIVE works for different experiment requirements. All the aforementioned functionalities may be tuned by parameters in the configuration file. A default value was also provided for these parameters, so that a user may run pyHIVE with a minimum parameters. 


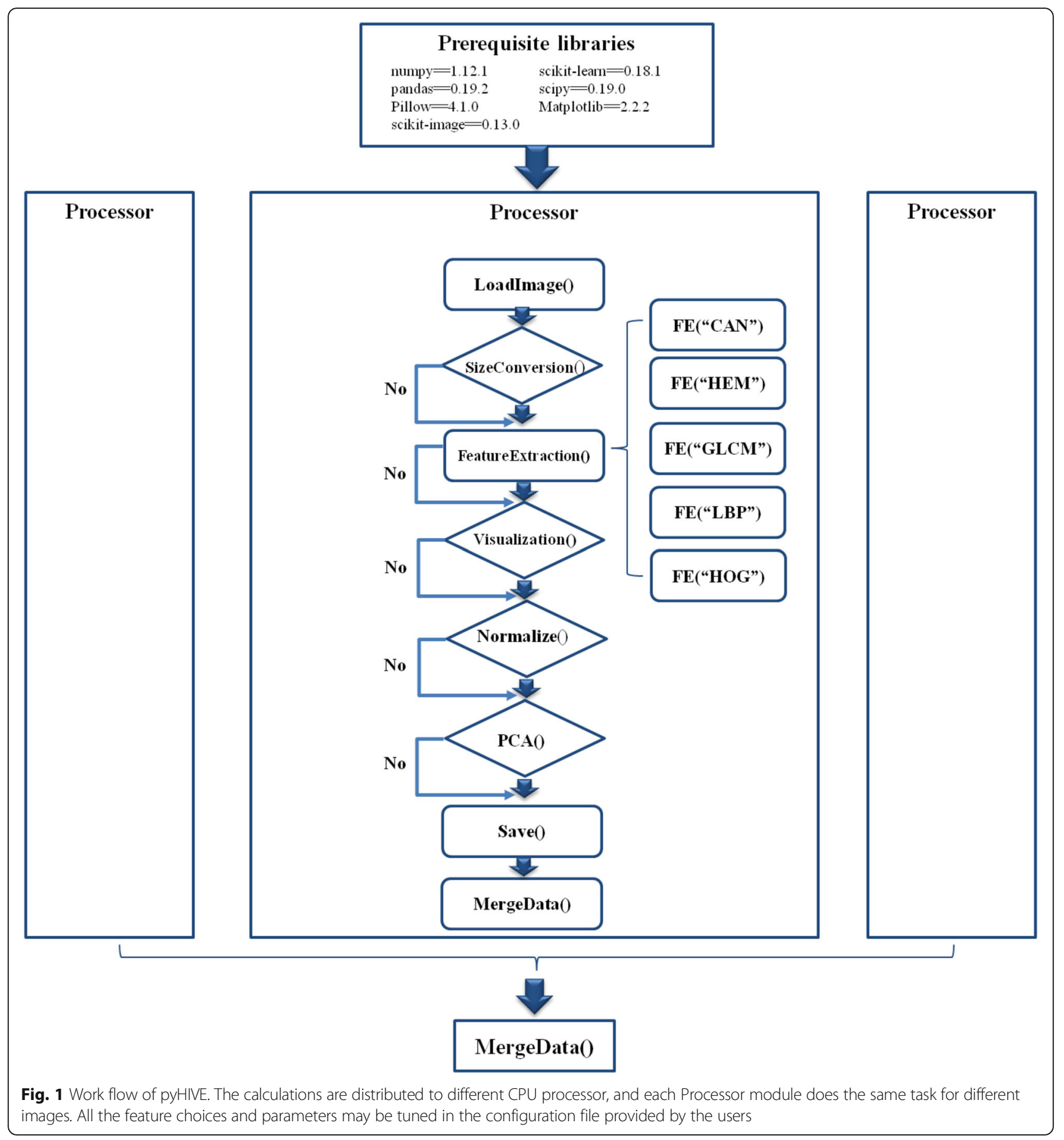

\section{Results}

We demonstrated how to use pyHIVE by a public dataset of gastrointestinal endoscopic images. The endoscopic images are publicly available at the El Salvador Atlas of Gastrointestinal Video Endoscopy, as similar in [12]. Detailed description of the dataset may be found in the section "Dataset details". Two feature extraction algorithms HOG and LBP were selected to investigate the above binary classification problem. HOG and LBP generated 95,256 and 84,480 features for each of the images, respectively. In order to avoid the over-fitting problem, the PCA module provided by pyHIVE was applied to the feature matrix. The default number of principal components was the smaller one of the two numbers of samples and features. So the number of features was reduced to $574(=243+331)$. Five representative classifiers, i.e. Support Vector Machine (SVM), Nearest Neighbor (NN), Decision Tree (DTree), Naïve 
Bayes (NBayes), Logistic Regression (LR), and Random Forest (RF), were chosen to evaluate the classification performance of a selected feature subset, as described in [15]. Accuracy was defined as the percentage of correctly predicted images, and the maximum accuracy (mAcc) of the five classifiers based on the given feature subset was defined as the performance measurement. All the classification performance measurements were calculated by the stratified k-fold cross validation strategy with the parameter $k=10$ by default.

After the preprocessing of PCA, both HOG and LBP features produced accurate classification models, as shown in Fig. 2. The feature scales of HOG and LBP are significantly different from each other. So they were processed using the normalization module provided by pyHIVE. HOG generated the best model with $\mathrm{mAcc}=$ 0.9896, and SVM performed the best among the five classifiers. It is interesting to observe that the second best model was achieved by LR with Accuracy $=0.9861$. The LBP features worked best with the classifier LR, and achieved $\mathrm{mAcc}=0.9862$. SVM didn't work well with the LBP features, and only achieved Accuracy= 0.5760 . A further improvement was achieved by combining features from both HOG and LBP, and the best model of $\mathrm{mAcc}=0.9914$ was achieved using the classifier LR. So generally, the classifier LR works very well with the image features generated by the HOG and LBP algorithms.

The classification performances of individual classes were evaluated for the best classifier LR, as shown in Fig. 3. The previous paragraph evaluated ten classifiers on three feature sets. So it's difficult to demonstrate all the confusion matrices and the prediction accuracies of the individual classes. The whole dataset of the features HOG+LBP was randomly split into the training (70\%) and test datasets. The confusion matrix of the best classifier LR was calculated by the 10 -fold cross validation strategy, as illustrated in Fig. 3. Both $\mathrm{P}$ and $\mathrm{N}$ classes were perfectly predicted, with sensitivity $=1.0000$ and specificity $=1.0000$. The experimental data suggested that besides the overall accuracy, the individual classes were also accurately predicted. Another classifier RF didn't perform as well as LR, and only achieved 0.9130 and 0.9417 for sensitivity and specificity, respectively.

Another evaluation experiment of pyHIVE was carried out on the two classes of canvas and cloth texture images from Contrib_TC_00000 of the Outex Texture Database [16]. Each class has 42 images and all the images are $256 \times 256$ in pixels. Two feature engineering algorithms GLCM and HEM were chosen for pyHIVE, and all the parameters used the default values. These two classes of texture images were significantly different from the endoscopic images, and may demonstrate pyHIVE's generalization on different image styles. A binary classification model was trained and evaluated, as described above. The classifier RF achieved worst accuracy 0.92 based on the GLCM features, while the other five classifiers achieved 1.00 in accuracy. All the classifiers except for LG and KNN achieved 1.00 in accuracy based on the HEM features. So the pyHIVE-generated image features perform well on the texture images, too.

The features of a given image file may be visualized as a histogram, as shown in Fig. 4. The algorithm HOG was chosen to calculate the features for two images 007744 and 007751 . The two images belong to the classes of canvas and cloth, respectively. So these two classes of texture images may be visually inspected for the differences between their HOG features. It is anticipatable to have a high accuracy for the binary classification model between these two classes of texture images.

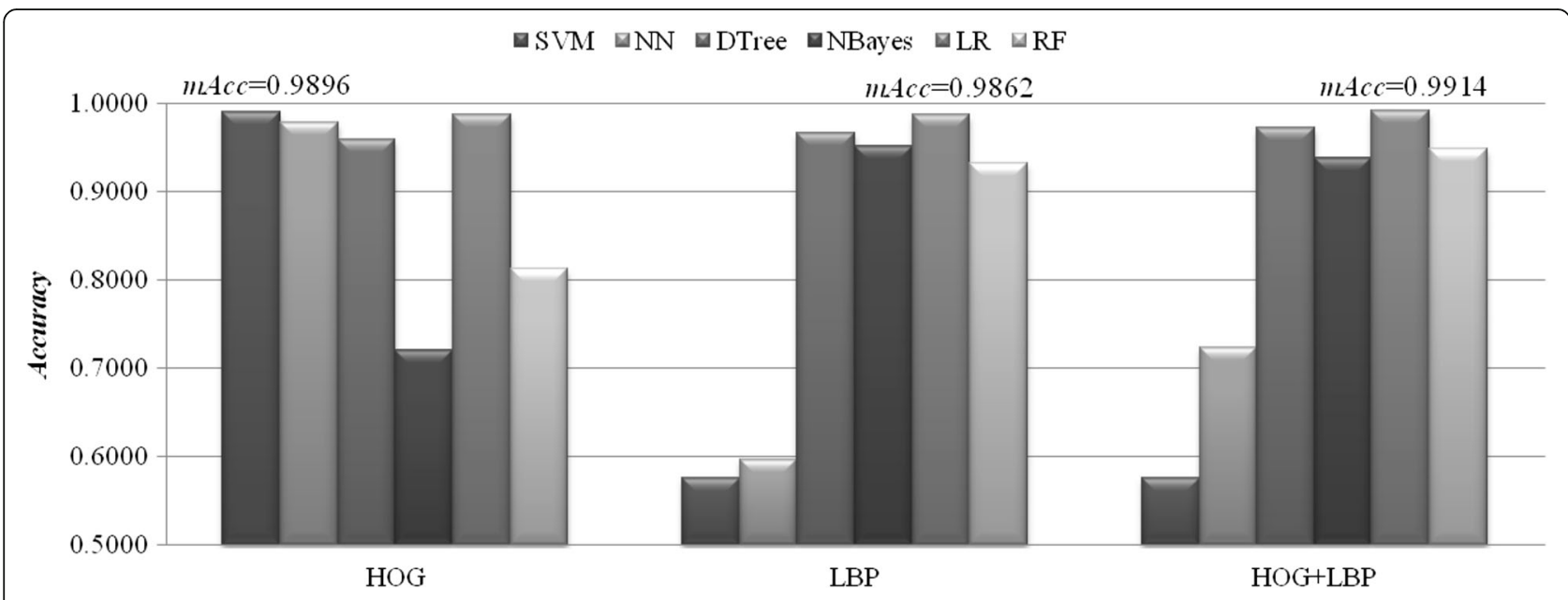

Fig. 2 Binary classification accuracy of the HOG and LBP image features generated by pyHIVE. mAcc is the maximum accuracy of the five classifiers 


\begin{tabular}{|c|r|r|r|}
\hline \multicolumn{2}{|c|}{ LR } & \multicolumn{2}{|c|}{ Predicted } \\
\cline { 2 - 4 } \multicolumn{2}{|c|}{ 10FCV } & $\mathrm{P}$ & $\mathrm{N}$ \\
\hline \multirow{2}{*}{ Real } & $\mathrm{P}$ & 103 & 0 \\
\cline { 2 - 4 } & $\mathrm{N}$ & 0 & 69 \\
\hline
\end{tabular}

Sensitivity $=\mathrm{TP} / \mathrm{P}=103 / 103=1.0000$ Specificity $=\mathrm{TN} / \mathrm{N}=69 / 69=1.0000$

\begin{tabular}{|c|r|r|r|}
\hline \multicolumn{2}{|c|}{ RF } & \multicolumn{2}{c|}{ Predicted } \\
\cline { 2 - 4 } \multicolumn{2}{|c|}{ 10FCV } & $\mathrm{P}$ & $\mathrm{N}$ \\
\hline \multirow{2}{*}{ Real } & $\mathrm{P}$ & 97 & 6 \\
\cline { 2 - 4 } & $\mathrm{N}$ & 6 & 63 \\
\hline
\end{tabular}

$$
\begin{aligned}
& \text { Sensitivity }=T P / P=97 / 103=0.9130 \\
& \text { Specificity }=T N / N=63 / 69=0.9417
\end{aligned}
$$

Fig. 3 Classification accuracies for the individual classes by the classifiers LR and RF. The left matrix was the confusion matrix of the 10-fold cross validation performances of the classifiers LR and RF. Sensitivity was the prediction accuracy of the positive samples, while the specificity was the accuracy of the negative samples

\section{Discussion}

This work proposed a biomedical-image feature extraction system pyHIVE, and an easy-to-use binary classification pipeline. Biomedical imaging technology produces huge amount of data, and requires specialized skills to read and analyze. The proposed system pyHIVE can generate a standard feature matrix from the biomedical images, so that a machine learning scientist may investigate the modeling part directly without the sophisticated imaging feature extraction knowledge. Five widely used image feature extraction algorithms were implemented and pyHIVE accepts $30+$ input image formats. The generated data matrix may be saved as one of the six standard file formats, i.e. CSV, PICKLE, JSON, EXCEL, TXT and SQL. To facilitate a quick prototyping investigation, a standard binary classification pipeline is also provided.
The parallelization performance of pyHIVE was tested in an Inspur Gene Server G100 with 256GB memory, 28 Intel $\mathrm{Xeon}^{\circ} \mathrm{CPU}$ cores $(2.4 \mathrm{GHz})$, and $26 \mathrm{~TB}$ hard disk. When pyHIVE ran under a single process, it spends $17.1667 \mathrm{~s}$ in extracting features from 574 images. When pyHIVE used 28 processes, only $1.2379 \mathrm{~s}$ was used to extract features from the aforementioned images. The result shows that the use of multi-cores computer can effectively reduce the time of image feature extraction, approximately 13.8676 times faster than the single-process version. So the parallelization module significantly increased the running speed of pyHIVE.

\section{Conclusions}

The python package pyHIVE proposed in this study is an easy-to-use biomedical image feature extraction system, and quite a few functional modules are also provided to
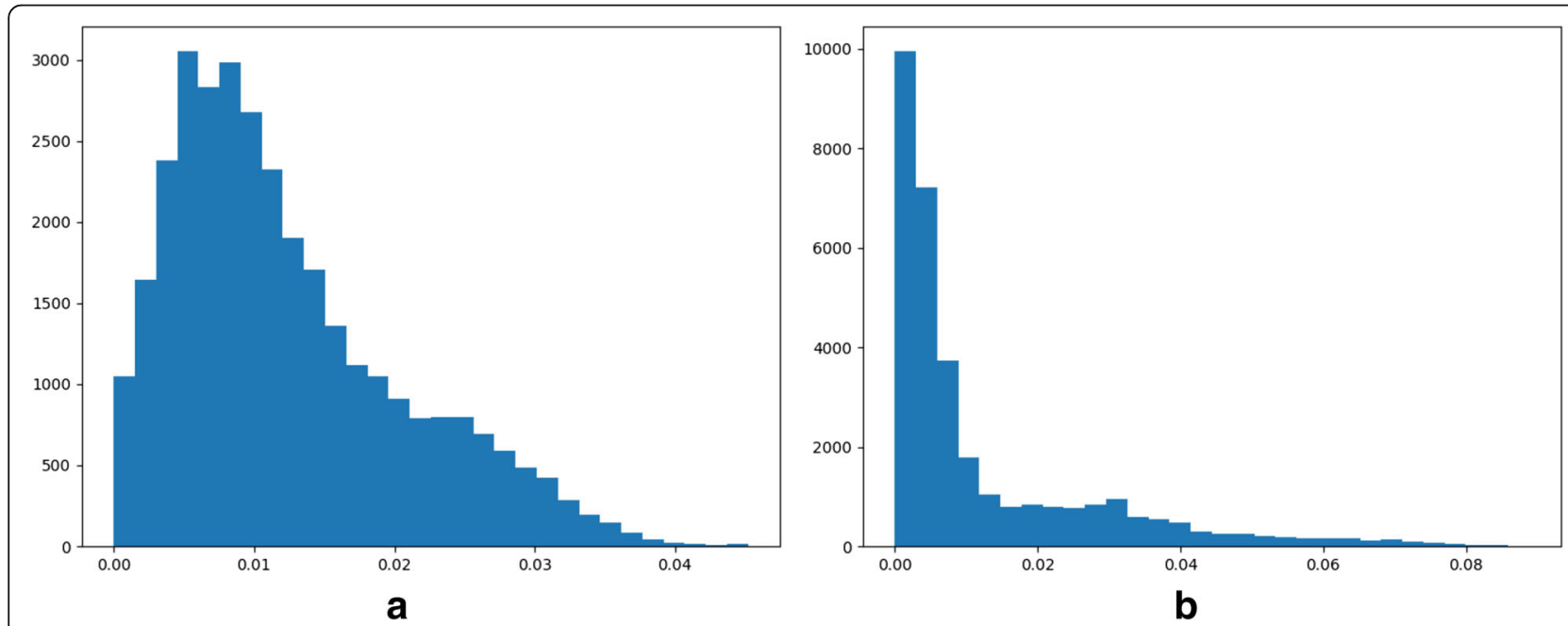

Fig. 4 Feature histograms calculated by pyHIVE. The non-normalized HOG features were calculated for (a) a canvas image 007744 and (b) a cloth image 007751 
facilitate a fast and intuitive exploration of an image based classification dataset. A large number of image file formats may be fed to pyHIVE, and the results may be exported as various standard file formats. pyHIVE is anticipated to help the biomedical image based research studies.

\section{Abbreviations}

CAN: Canny; DTree: Decision Tree; GLCM: Gray-level co-occurrence matrix HEM: Hessian Matrix; HOG: Histogram of oriented gradients; LBP: Local binary pattern; LR: Logistic Regression; mAcc: Maximum accuracy; NBayes: Naïve Bayes; NN: Nearest Neighbor; PCA: Principal Component Analysis; pyHIVE: Python-based Health-related Image Visualization and Engineering system; RF: Random Forest; SVM: Support Vector Machine

\section{Acknowledgements}

The insightful and constructive comments from the anonymous reviewer are greatly appreciated.

\section{Funding}

This work was supported by the Strategic Priority Research Program of the Chinese Academy of Sciences (XDB13040400), Jilin Provincial Key Laboratory of Big Data Intelligent Computing (20180622002JC), the Education Department of Jilin Province (JJKH20180145KJ), and the startup grant of the Jilin University. This work was also partially supported by the Bioknow MedAl Institute (BMCPP-2018-001) and the High Performance Computing Center of Jilin University, China.

\section{Availability of data and materials}

The system pyHIVE and the demonstrative example are freely available and maintained at http://www.healthinformaticslab.org/supp/resources.php

\section{Authors' contributions}

FZ and RZhang conceived and designed the project. RZhang and RZhao discussed and designed the pyHIVE modules. RZhang implemented the whole system. RZhang, RZhao, XZ, DW, WZ and XF carried out the experiments and discussed the data. RZhang, WZ, and XF drafted the manuscript. FZ revised and polished the manuscript. All authors read and approved the final manuscript.

\section{Ethics approval and consent to participate}

Not applicable.

\section{Consent for publication}

Not applicable.

\section{Competing interests}

The authors declare that they have no competing interests.

\section{Publisher's Note}

Springer Nature remains neutral with regard to jurisdictional claims in published maps and institutional affiliations.

\section{Author details \\ 'BioKnow Health Informatics Lab, College of Computer Science and Technology, and Key Laboratory of Symbolic Computation and Knowledge Engineering of Ministry of Education, Jilin University, Changchun 130012, Jilin, China. ${ }^{2}$ Bioknow Health Informatics Lab, College of Software, Jilin University, Changchun 130012, Jilin, China.}

Received: 29 December 2017 Accepted: 9 November 2018 Published online: 26 November 2018

\section{References}

1. Nair M, Sandhu SS, Sharma AK. Prognostic and predictive biomarkers in Cancer. Curr Cancer Drug Targets. 2014;14(5):477-504.

2. Vlaisavljevich $E_{1}$ Owens $G$, Lundt J, Teofilovic D, Ives K, Duryea A, Bertolina J, Welling TH, Xu Z. Non-invasive liver ablation using histotripsy: preclinical safety study in an in vivo porcine model. Ultrasound Med Biol. 2017;43(6): 1237-51.

3. Sharma S. Translational multimodality neuroimaging. Curr Drug Targets. 2017:18(9):1039-50

4. Yang D, Subramanian G, Duan J, Gao S, Bai L, Chandramohanadas R, Ai Y. A portable image-based cytometer for rapid malaria detection and quantification. PLoS One. 2017;12(6):e0179161.

5. Ding Y, Pardon MC, Agostini A, Faas H, Duan J, Ward WOC, Easton F, Auer D, Bai L. Novel methods for microglia segmentation, feature extraction, and classification. IEEE/ACM transactions on computational biology and bioinformatics. 2017:14(6):1366-77.

6. Ding Y, Ward WO, Duan J, Auer DP, Gowland P, Bai L. Retinal vasculature classification using novel multifractal features. Phys Med Biol. 2015;60(21): 8365-79.

7. Adetiba E, Olugbara OO. Lung cancer prediction using neural network ensemble with histogram of oriented gradient genomic features. Sci World J. 2015;2015:786013.

8. Unay D, Ekin A, Cetin M, Jasinschi R, Ercil A. Robustness of local binary patterns in brain MR image analysis. In: Engineering in Medicine and Biology Society, 2007 EMBS 2007, Lyon, France, 29th Annual International Conference of the IEEE. IEEE; 2007. p. 2098-101.

9. Nanni L, Lumini A, Brahnam S. Local binary patterns variants as texture descriptors for medical image analysis. Artif Intell Med. 2010;49(2):117-25.

10. Wang Y, Zhang Y, Yao Z, Zhao R, Zhou F. Machine learning based detection of age-related macular degeneration (AMD) and diabetic macular edema (DME) from optical coherence tomography (OCT) images. Biomed Opt Express. 2016;7(12):4928-40.

11. Vallieres M, Freeman C, Skamene S, El Naqa I. WE-C-WAB-02: joint FDG-PET/ MR imaging for the early prediction of tumor outcomes. Med Phys. 2013; 40(6Part29):477.

12. Tursi T. Use of ss-blocker therapy to prevent primary bleeding of esophageal varices. J Am Acad Nurse Pract. 2010;22(12):640-7.

13. Tang Z, Zhang J, Gui W. Selective search and intensity context based retina vessel image segmentation. J Med Syst. 2017;41(3):47.

14. Yang D, Wang X, Duan Y, Tan J, Mutic S. SU-E-J-76: 3D soft tissue boundary detection for automatic verification of deformable image registration. Med Phys. 2013;40(6Part7):167.

15. Ge R, Zhou M, Luo Y, Meng Q, Mai G, Ma D, Wang G, Zhou F. McTwo: a two-step feature selection algorithm based on maximal information coefficient. BMC Bioinformatics. 2016;17:142

16. Ojala T, Maenpaa T, Pietikainen M, Viertola J, Kyllonen J, Huovinen S. Outex new framework for empirical evaluation of texture analysis algorithms. In: international conference on pattern recognition; 2002. p. 701-6.

\section{Ready to submit your research? Choose BMC and benefit from:}

- fast, convenient online submission

- thorough peer review by experienced researchers in your field

- rapid publication on acceptance

- support for research data, including large and complex data types

- gold Open Access which fosters wider collaboration and increased citations

- maximum visibility for your research: over $100 \mathrm{M}$ website views per year

At $B M C$, research is always in progress.

Learn more biomedcentral.com/submissions 